\title{
AUTONOMOUS ROBOTIC INSPECTION IN TUNNELS
}

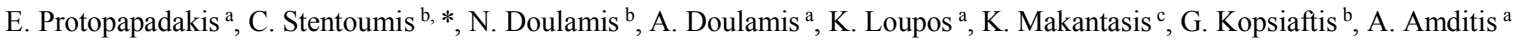 \\ ${ }^{a}$ Institute of Communication and Computer Systems, Zografou GR-157 80, Athens, Greece - \\ \{eprotopapadakis, a.amditis, kloupos\}@iccs.gr, doulamisanastasios@gmail.com \\ ${ }^{\mathrm{b}}$ Lab. of Photogrammetry, National Technical University of Athens, GR-15780, Greece - \{cstent, ndoulam, gkopsiaf\}@mail.ntua.gr \\ c Tech. Univ. of Crete, Chania, Greece - konst.makantasis@gmail.com
}

Commission V, WG V/5

KEY WORDS: Inspection, Robotics, Automation, Deep Learning, Scanner, Structural Assessment, Defects, Reconstruction

\begin{abstract}
:
In this paper, an automatic robotic inspector for tunnel assessment is presented. The proposed platform is able to autonomously navigate within the civil infrastructures, grab stereo images and process/analyse them, in order to identify defect types. At first, there is the crack detection via deep learning approaches. Then, a detailed 3D model of the cracked area is created, utilizing photogrammetric methods. Finally, a laser profiling of the tunnel's lining, for a narrow region close to detected crack is performed; allowing for the deduction of potential deformations. The robotic platform consists of an autonomous mobile vehicle; a crane arm, guided by the computer vision-based crack detector, carrying ultrasound sensors, the stereo cameras and the laser scanner. Visual inspection is based on convolutional neural networks, which support the creation of high-level discriminative features for complex non-linear pattern classification. Then, real-time 3D information is accurately calculated and the crack position and orientation is passed to the robotic platform. The entire system has been evaluated in railway and road tunnels, i.e. in Egnatia Highway and London underground infrastructure.
\end{abstract}

\section{INTRODUCTION}

Inspection and maintenance of transportation tunnels is a challenging and demanding process, due to the complex surveillance conditions and large scale requirements. Due to tunnel ageing, their efficient inspection has become a topic of great concern in engineering. There are several factors affecting the health of a tunnel and these factors should be efficiently and effectively monitored and assessed in order the engineers to understand the reasons of a structure degradation (Sandrone and Labiouse, 2011; Yuan et al., 2012).

Continuous inspection of the parameters (e.g., cracks, defections, deformations) that result in a deterioration of the tunnel infrastructure is an arduous and time consuming process (Victores et al., 2011; Yoon et al., 2009). Usually, inspection processes include scanning of the tunnel intrados for potential defects, such as cracks, opening of joints, concrete corrosion or spall, or deformations of the curved tunnel surface, which could have a serious impact on the tunnel stability.

Currently inspection and maintenance of a tunnel relies on manual visual monitoring, a process that it has several drawbacks. Apart from being costly and time consuming, it is highly depended on the human subjectivity, resulting, consequently in inaccuracies mainly regarding the positioning and the type of the problem. For this reason, several approaches have been proposed in the literature to increase the costefficiency in inspection through automation (Krisada, 2014).

In this context, the European Union has funded a research project with the main target of providing automated solutions for tunnel inspection and monitoring by integrating a computer vision methods, photogrammetry and robotic technology. The presented work is a part an intelligent platform, with the focus on developing an integrated autonomous robotic system, which utilizes deep machine learning architectures and intelligent control tools to automatically detect tunnel defects and provide structural engineers with sufficient data to evaluate the stability of underground infrastructures and curry out the required maintenance procedures.

\subsection{Related work}

In order to implement an autonomous robotic tunnel inspection platform, the following issues should be addressed: (a) nondestructive assessment approaches (NDAs), (b) navigation platform and (c) communication among robotic parts. The majority of the NDA exploits RGB image based techniques. Since the entire inspection scheme is based on the assessment performance, NDA is considered as core technological tool of the presented research.

In this context the work of Sinha and Fieguth (2006) develops a two-step algorithm to extract features and in the sequel to identify cracks in pipes. The presented approach exploits statistical filtering. The work of Paar et al. (2006) imitates a region expansion technique to detect and then to follow cracks. The architecture exploits a set of images that are unfolded on the theoretical surface of the tunnel intrados. Similarly, Lee et al. (2007) exploited image processing algorithms to detect and visually categorize cracks depending on surface analysis and pattern recognition methods. The whole system has been embedded on a robotic platform with the main purpose of taking images and, in the sequel, identify and measure the cracks. Intensity features and Support Vector Machines (SVMs) for crack detections on tunnel surfaces where used in Liu et al. (2002).

\footnotetext{
* Corresponding author
} 
On the one hand, there is a two-step approach; feature extraction and identification (i.e. classification via machine learning approaches). Such approaches involve colour properties, different non-RGB colour spaces and various machine learning algorithms, edge detection techniques, Abdel-Qader et al. (2003) and graph based search algorithms, Yu et al. (2007). . The exploitation of more sophisticated features has also been proposed. Histogram of Oriented Gradient (HOGs) features and SVMs are utilized in the work of Halfawy and Hengmeechai (2014).. Shape-based filtering is another approach, exploited in the work of Jahanshahi et al. (2013) for crack detection and quantification.

On the other hand we have the Convolutional Neural Networks (CNNs), which extract the most appropriate features, given a classifier. The work of Makantasis et al. (2015) exploit CNN to hierarchically construct high-level features, describing the defects, and a Multi-Layer Perceptron (MLP) that carries out the defect detection task in tunnels. Such an approach offers an automated feature extraction and adaptability to the defect type(s. Latter, Protopapadakis and Doulamis (2015) further improved the aforementioned approach, by incorporating a prior, unsupervised, image annotation mechanism to facilitate the initialization steps (i.e. training data creation) of the $\mathrm{CNN}$.

Stent et al. (2013) reconstruct the lining of a tunnel based on the prior knowledge of tunnel geometry. This model is then updated by new images in order to detect changes and defects. Apart from image-based systems, 3 D laser scanners, either time-of-flight, or phase-shift scanners have been tested for tunnel inspection (Fekete et al., 2010; Yoon et al., 2009; Yu et al., 2007; Yuan et al., 2012). The obtained data are processed off line and the 3D structure of the tunnel is reconstructed. However, the complete scan of kilometres of tunnelling results in huge amounts of data, which in the sequel are difficult to be manipulated. However, the advantages of such approaches is that they are not sensitive on the low lighting conditions of the tunnel and direct processing of the 3D point clouds results in extraction of features that can be used for a more efficient monitoring of tunnels' conditions in contrast with the traditional image-based approaches (Sandrone, 2013; Yoon et al., 2009).

Integrated methods also appeared (Victores et al., 2011), where a robot is presented for correcting lining anomalies. Recently, (Sandrone, 2013) introduces a framework for condition tunnel assessment of Swiss railways. Defects are detected by surveying the lining with a laser scanner. However, in this case data are processed off-line. The system incorporates point cloud from laser scanner and thermal data processing in order to detect and identify specific anomalies.

To sum up, tunnel inspection is a growing field for automation. Some work has been done in the past, but in an unstructured way missing real conditions. Our key difference is that our work is guided by the direct needs of structural engineers, hence the width of the cracks in quest, which are the tiniest of the defects, is significantly smaller (sub-millimetre) and the scale of the problem larger by order of magnitude, when compared to most relevant publications. Furthermore, real tests are missing and no testimony of accuracy is provided. Finally, large road tunnels are much wider in size than railways and are an unconstrained environment, thus more difficult to document and process due to much more data.

\subsection{Contribution}

This paper extends the current state of the art by proposing an integrated platform for automatic inspection of road and railway tunnels. The method incorporates advantages in computer vision, photogrammetry and robotic technology in order to improve the conditional tunnel assessment and the automation. Initially, a crack detector is implemented exploiting state-of-the-art computer vision and machine learning tools. This is performed via combining low level image processing methods and nonlinear deep machine learning algorithms. The latter, better emulates humans' brain activity in understanding complex nonlinear patterns as shown in Hinton et al. (1995). Deep learning propagates the obtained information (i.e. the image patches) via a predefined hierarchical structure, constructing high levelfeatures. Such process offers significant advantages in information organization, classification and performance, compared to other learning paradigms, like conventional neural networks and SVMs.

A crack can appear for many reasons, varying from rapid drying to load disorders. In order to identify correctly what caused the crack generation, the detected regions and the area in close proximity are scanned using a laser scanner. Then, the extracted point clouds are modelled though simplified geometrical surfaces by applying a two-step non-linear least square algorithm; (a) transformation of the detected point clouds from the arbitrarily coordination system of the laser scanner to a given one, where tunnel surface can be easily modelled; (b) fitness of the tunnel surface, as provided by the civil engineers during survey, to the transformed point clouds. This way, the system can provide information about the tunnel structure deformations that are mainly responsible for generating the cracks.

Image based 3D reconstruction methods, via customized dense matching algorithms, that take into consideration the geometry of the tunnel, were also exploited. Such module was used for extracting with high accuracy the 3D measurements of particular points in the image in order to allow navigation of the robotic arm. This is considered as a necessary step for the robotic platform so as to take local measurements regarding crack properties and geometric characteristics of very high precision.

The rest of this paper is organized as follows: Section 2 presents the robotic platform and analyses the different components of the architecture. Section 3 describes the computer vision algorithm used for defects and cracks detection onto the 2D image plane. Section 4 discusses how a detailed 3D model of the crack surface is derived employing photogrammetric methods, while Section 5 describes how a detailed 3D model of a slice of the tunnel is taken from laser scanners to measure deformations. Finally, conclusions are given in Section 6.

\section{THE ROBOTIC PLATFORM}

Figure 1 shows a block diagram of the proposed architecture. In particular, the system consists of a mobile vehicle which is autonomously moving within the indoor tunnel structure. The platform is equipped with the robotic arm that is used to touch a crack and then take measurements regarding its nature using ultrasound devices. Visual cameras are mounted on the platform and then computer vision algorithms are applied to detect cracks and defects onto the 2D image plane. This is a very arduous task due to the bad illumination conditions encountered in the tunnel, and the existence of many visual effects like shallow water erosions, paintings removals, etc. that should not be confused with defects and cracks. 
The mounted camera system is also used to track the detected crack so as to navigate the robotic arm to touch the crack and take precise measurements. Finally, a detailed 3D model of the crack is derived using photogrammetric methods while a $3 \mathrm{D}$ model of a tunnel slice is created employing a laser scanner to measure deformations. A visual depiction of the robotic platform is shown in Figure 2. This figure presents both the autonomous vehicle and the robotic arm, while simultaneously illustrates the camera and the laser scanner sub-systems of our robot.

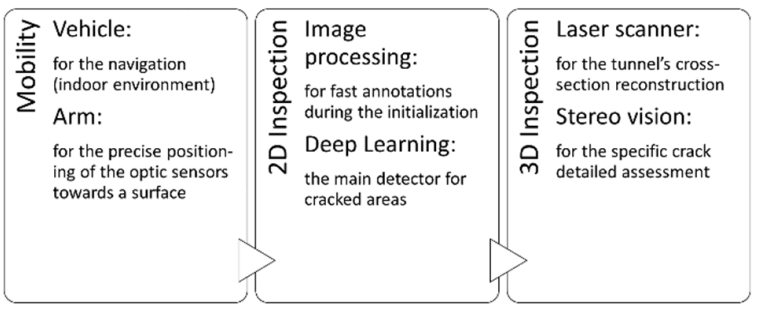

Figure 1. The robotic platform task description.

\subsection{Mobile Vehicle and Crane}

The main platform is a wheeled vehicle, which is retrofitted with robotic sensors. It, also, includes an extendable crane system able to extend to up to 11 meters high (lengths commonly found in tunnel environments) and has a robotic manipulator (tip) attached to its end. The movement of all systems is automated through the usage of the respective robotic controllers and supported by the Integrated Global Controlling (IGC) system that is also responsible for the overall system operation and mission execution.

The global controller accepts various inputs. Among them are the position of crack (computer vision output), semantic information on the state of the system and the required action/behaviour, laser status (the one that creates a 3D representation of the local area and tunnel wall), the robotic tip trajectory estimations and other sensing information.

The robotic vehicle is equipped with additional laser systems for safety and navigation purposes. On the vehicle, also, are energy modules for the supply of the whole system. There are also appropriate modules and connection mechanisms for the communication physical interface. With this equipment onboard, the robotic vehicle is able to execute missions on robotic inspection of tunnel linings in an autonomous manner. A description of the main robotic tasks are shown in Figure 3.
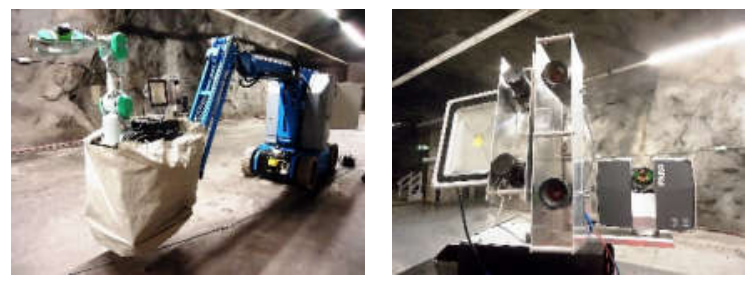

Figure 2. Images of the first functional prototype robotic vehicle for tunnel inspection. Left image: an overall image of the autonomous vehicle, the robotic arm and the imaging system; right image: the robot's sub-system of sensors, the cameras and the laser scanner.
The system operation includes the robotic vehicle, rolling at low speed inside the tunnel (length), with the computer vision system continuously taking images of the tunnel walls. As soon as the computer vision detects any crack on the tunnel wall (in real time), the controller commands the robot to stop and perform a detailed inspection; this includes the 3D modelling of the surrounding (to the crack) area, a laser profiling of the tunnel cross-section and the positioning of the robotic arm at a suitable position so as precise crack width and depth ultra-sonic measurement can be performed.

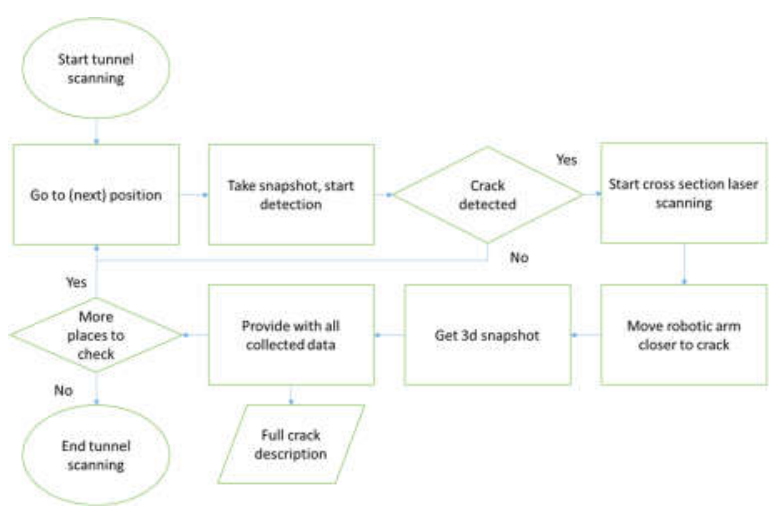

Figure 3. The robotic platform task description.

As soon as all measurements were concluded, the robotic arm returns to its "home" position and the inspection continues until the next crack is detected. All measurements and defect information are also processed off-line for a series of other defects (e.g. spalling, delamination etc.) and also processed by a decision support system that characterises the severity of the damage and acting as the user interface of the tunnel operators/inspectors.

\subsection{Robotic Arm}

The robotic arm, mounted on the crane end, is a Mitsubishi PA$10 \mathrm{arm}$, together with a servo driver controller. The arm movement is able to cover from few centimetres to one meter approximately. The arm has six degrees of freedom and can thus cover all the possible geometry of any fissure. The control software is based on the ROS (Robot Operating System) architecture (just like the mobile vehicle) and the internal modules communicate using YARP (Yet Another Robotic Platform - Meta et al., 2006).

The robotic arm also uses a $2 \mathrm{D}$ range laser to compute trajectories during the inspection process mounted on the arm. The ultrasonic sensors are fixed to the tip of the arm so they can be directed to the exact position of measurement for direct measurement of the identified crack width and depth. The position of the crack is identified by the vision system as described below.

\subsection{Vision System}

The cameras along with the laser scanner are the sensors of the integrated surveying and recognition system. Two sets of stereocameras are used for grabbing the necessary images. The cameras are synchronized through the Arduino Uno board, which serves as a trigger/ pulse generator. The first stereo pair is responsible for the crack and the other defects detection that lay on the tunnel lining; it operates in real-time from a distance of two to four meters and it extracts the $3 \mathrm{D}$ information, which is necessary to 
the robot in order to place the ultrasonic sensor to the correct position for depth measurements.

The second stereo pair is used for the full $3 \mathrm{D}$ reconstruction of high fidelity models of the areas of cracks. This setup regards the road tunnels, which have a large width (over $9 \mathrm{~m}$ diameter); in the cases of railway tunnels (3.5m diameter) all four cameras form an array that can cover larger portions of the tunnel lining in each pass. Table Table 1 presents the technical specifications of the used hardware. It should be noticed that, while the results presented in this paper are definitely positive and promising for the automation in tunnel inspections, the hardware needed is still under investigation, as the discussed prototype is not, of course, ready for production. Thus, more suitable, from cost -benefit point of view, sensors configurations could exist.

\subsection{D Laser Scanner}

The FARO 3D laser scanner (Table Table 2) has been mounted on the robotic platform. This is being used for a precise calculation of any tunnel deformation at positions where a deformation could be present (i.e. at positions of cracks). This system is activated only at the positions of cracks detected by the computer vision system. The information are then passed to the decision support system for positioning and visualization purposes (user interfacing etc.). The laser system is able to operate with an accuracy of $2 \mathrm{~mm}$.

\begin{tabular}{ll}
\hline Model & Point Grey - GS3-U3-91S6C-C \\
Number of pixels & $9.1 \mathrm{Mega}$ Pixels \\
Pixel / Sensor size & $3.69 \mu \mathrm{m} / 3376$ x 2704 pixels \\
Focal length & $12.5 \mathrm{~mm} / 25 \mathrm{~mm}$ \\
FoV cf / CCD diagonal & $1.7 / 1 "$ \\
Sensor type & Sony ICX814, Global shutter \\
Weight & $2 \times\{90 \mathrm{~g}($ sensor $)+290 \mathrm{~g}($ lens $)\}$ \\
Single Camera size & $44 \times 29 \times 58 \mathrm{~mm}$ (sensor) $+120 \times 90$ \\
\hline
\end{tabular}

Table 1. Stereo camera technical specifications.

\begin{tabular}{ll}
\hline Model & Faro Focus 3D X 130 \\
Scan density & $<1 \mathrm{~mm}$ \\
Error & $2 \mathrm{~mm} @ 50 \mathrm{~m}$ \\
Noise & $0.3 \mathrm{~mm}$ \\
Beam diameter & $3 \mathrm{~mm} @$ exit \\
Weight & $5 \mathrm{~kg}$ \\
Dimensions & $240 \times 200 \times 100 \mathrm{~mm}$ \\
\hline
\end{tabular}

Table 2. 3D laser scanner technical specifications.

\subsection{Information Exchange / Integration}

The different modules of the computer vision and the photogrammetry sub-system communicate among each other and with the Intelligent Global Controller of the autonomous platform with the YARP open-source middleware. YARP consists of libraries, protocols, and tools, designed to control and manage multiple devices and modules.

During the operation of YAPR a message is sent over the communication buss channel of the robotic system. This message is passing though out the different modules of the robotic systems and sensors. Only the sensor that has been assigned to this port and to this interface are allowed to interact with the message. The selection of YARP was made due to the straightforward implementation and the ability to easily set it up in Linux and Windows OS, in contrast to other Robot Operating Systems.

\section{2D INSPECTION}

We consider the detection of concrete defects in tunnels using monocular camera's RGB images. Seen as an image segmentation problem, the detection of defects entails into a classification problem. Each one of the image's pixels belong in one out of two classes; defection class and no defection class. Visual inspection is mainly based on deep learning approaches. In particular, CNNs are utilized for both defect recognition and crack identification. The innovation of such approach can be summarized in three main points; the automated feature extraction, adjustability to the defect type(s), and no need for special set-up for the image acquisition (e.g., specific cameraobject distance).

CNNs hierarchically construct high-level features, and a MultiLayer Perceptron (MLP) carries out the defect detection task. Defects are described from both visual and special information. Visual information is derived using the RGB values of each pixel. Spatial information is obtained by taking into consideration a neighbourhood around each pixel.

For any RGB image, the presented method creates a new binary image annotating the two possible classes; defect or non-defect, named positive $(\mathrm{P})$ and negative $(\mathrm{N})$ class, respectively. A comparison between actual ground truth and model annotated images results in the confusion table creation; A $2 \times 2$ matrix that reports the number of false positives (FP), false negatives $(\mathrm{FN})$, true positives (TP), and true negatives (TN).

Given these values we are able to calculate various performance metrics regarding the defect detection performance. Calculated metrics are: sensitivity (TPR), specificity (SPC), precision (PPV), negative predicted value (NPV), false positive rate (FPR), false discovery rate (FDR), miss rate (FNR), accuracy (ACC) and F1 score (F1). Metrics of special interest are: Sensitivity (proportional to TP) and miss rate (proportional to $\mathrm{FN}$ ), which are both strongly connected to defect detection.

\subsection{Defect Recognition}

The defect recognition follows the pipeline of Makantasis et al. (2015). This is presented in Figure 4. High-level feature construction, through the $\mathrm{CNN}$, is based on low-lever features, such as: edges, frequency, entropy, texture, etc. A typical output of such mechanism is shown in Figure 5.

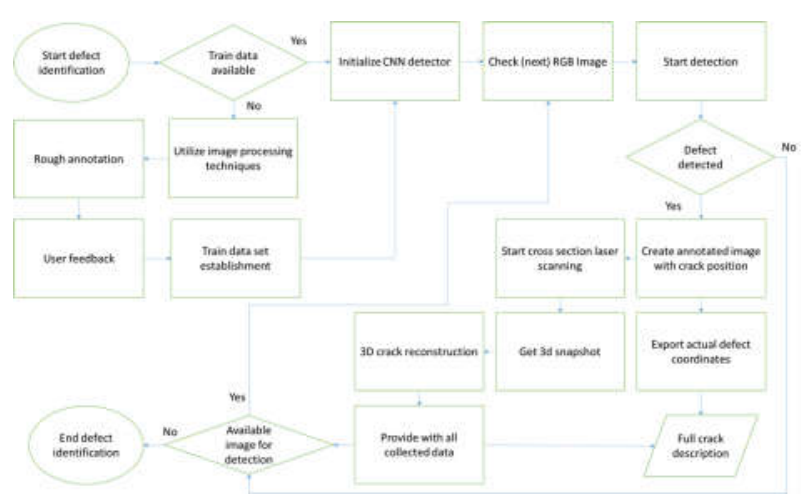

Figure 4. The defect identification approach based on RGB images and laser scanning. 


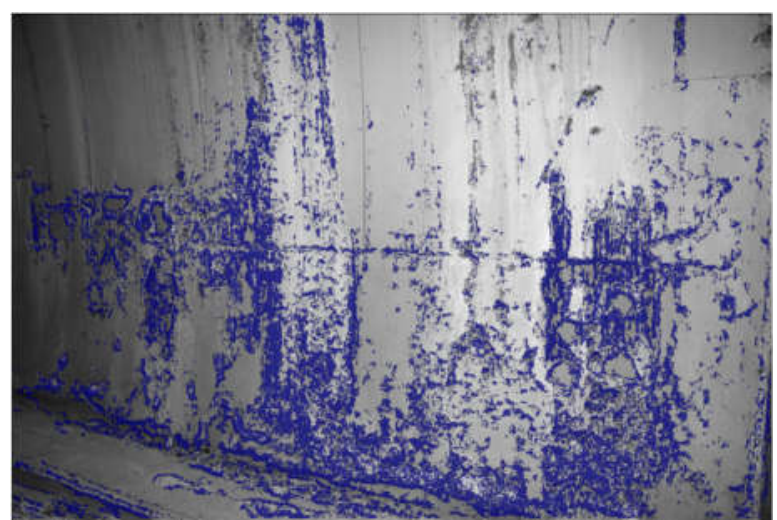

Figure 5. Areas with any type of defect are marked with blue dots.

\subsection{Crack Identification}

The crack identification module of the described system was implemented was based on the work of Makantasis et al. (2015); on CNNs. Yet, in order to facilitate the data set creation, we employ an image processing technique as in Protopapadakis and Doulamis (2015).

That technique exploits: the intensity of pixels and their spatial relations, morphological operations and filtering schemes. Such an approach does not require annotated data incorporates a prior, image processing, detection mechanism. Such mechanism stands as a simple detector and is only used at the beginning of the inspection. Possible defects are annotated and then validated by an expert; after validating few samples, the required training dataset for the deep learning approach has been formed (Figure $6)$.

The crack detection is based solely on the CNN. The input of the $\mathrm{CNN}$ are patches of dimensions $9 \times 9$, in order to take into consideration the closest 24 neighbours of each pixel. The first layer of the proposed $\mathrm{CNN}$ is a convolutional layer with $C_{1}=15$ trainable filters of dimensions $5 \times 5$. Due to the fact that we do not employ a max pooling layer, the output of the first convolutional layer is fed directly to the second convolutional layer ( 30 kernels of size $3 \times 3$ ). Then, the third layer ( 45 kernels of size $3 \times 3$ ) creates the input vectors for the MLP.
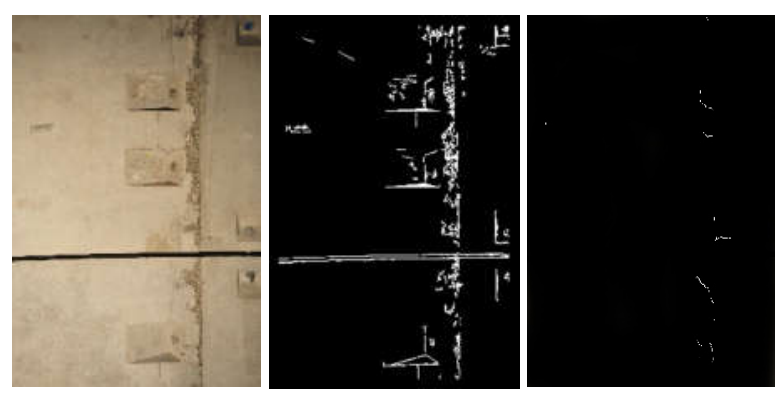

Figure 6. Left: original image; middle: possible defect areas presented for validation; right: $\mathrm{CNN}$ output after training.

Once the final annotation is concluded the assessment mechanism is activated in order to describe located cracks, i.e. calculate the length, width and orientation over the wall. Minimum bounding boxes are calculated for all cracked areas above a predefined threshold. Then, for every bounding box, random pixels, depicting a crack, are uniformly selected. These pixels can provide an accurate description of the crack and reduce the processing of the quantification process.

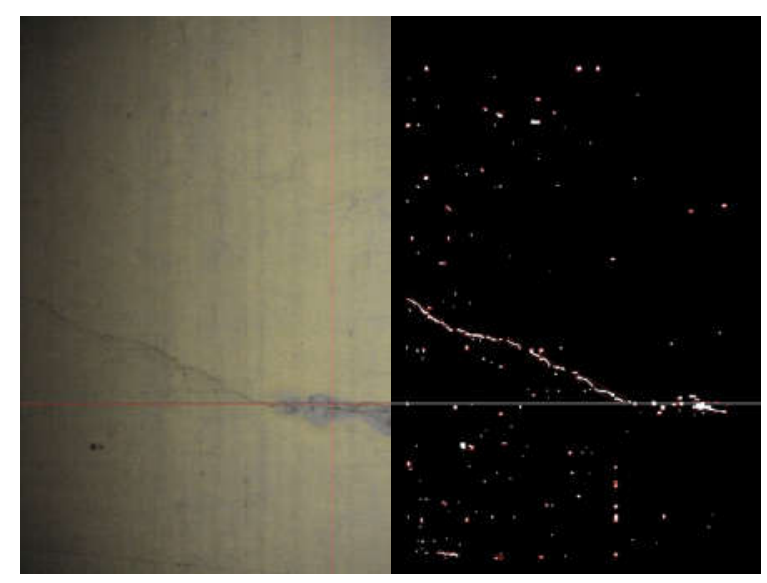

Figure 7. Illustration of the original image and the assessment over the CNN annotated one. The red bounding boxes depict the regions under inspection. The robotic arm will then move to a maximum-crack-width point, indicated by '+', for further investigation.

\section{3D INSPECTION}

As soon as a crack, described critical from the structural integrity point of view, is detected the inspection process moves towards the $3 \mathrm{D}$ world space. For this purpose, the two stereo-cameras are exploited in a twofold manner: the real-time 3D extrapolation of a crack and the full 3D reconstruction of a high fidelity model of the wider area of a crack.

The stereo-cameras are calibrated at the beginning of the inspection process with the use of a chessboard pattern (Douskos et al., 2009). Prokos et al. (2009) show that stereo cameras calibration is feasible from a chessboard pattern without visible edges; which is the discussed case since the system cameras are calibrated from $1.5 \mathrm{~m}$ and $3 \mathrm{~m}$ with the same chessboard plane, thus it is only partial visible from $1.5 \mathrm{~m}$.

After this step, the terrestrial laser scanner is registered to the image data and the mounting frame of the cameras through an initial rigid body transformation (Arun et al., 1987) and a subsequent more precise least-square fit (Grammatikopoulos et al., 2015) with the help of the chessboard. The subsequent stereomatching and $3 \mathrm{D}$ reconstruction processes are executed on the rectified epipolar images.

\subsection{Real-time 3D extraction for robot guidance}

The recognition algorithms and 3D information extraction algorithm are combined as a real-time system, which performs an accurate calculation of the crack position world coordinates and pass this information to the robotic platform; this is necessary in order to place the ultrasonic sensor on the actual tunnel crack and take the appropriate stereo-images for modelling of full and high fidelity. The thickest part of a crack is identified and an area around it is reconstructed. The area is reconstructed in order to evaluate the correctness of the extracted crack position, based on the average depth of the smooth tunnel surface Figure 8. 

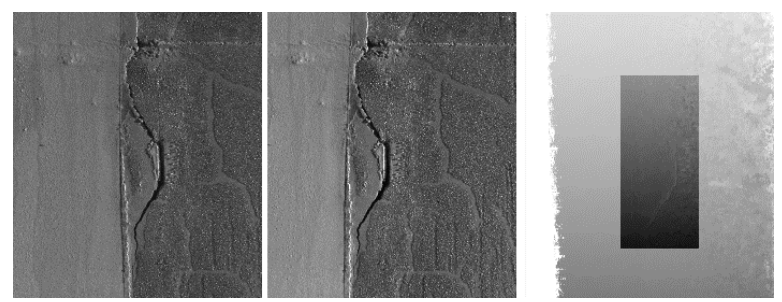

Figure 8 . Detection and 3D extrapolation of a crack $(3 \mathrm{~mm}$ wide). $1^{\text {st }}$ and $2^{\text {nd }}$ images are the stereo-pair; $3^{\text {rd }}$ image: the estimated disparity map of the left image with the centre area stretched in order to visualize the crack.

\subsection{D Reconstruction for Visual Inspection by an Expert User}

High fidelity 3D models are also produced and served to the expert decision support system (DSS) that is responsible for the risk assessment of the tunnel condition. It is also available to the tunnel maintenance inspectors for quality visual observation through the human DSS interface. For this purpose, three stereoimages of the detected crack are grabbed from pre-defined positions to ensure the completeness of the reconstructed model. The 3D models are reconstructed offline with high accuracy $(\sim 1 \mathrm{~mm})$ and resolution $(<1 \mathrm{~mm})$.

The reconstruction pipeline is based on a fusion of an adaptive local matching algorithm (Stentoumis et al., 2014) and semiglobal matching (Hirschmüller, 2008). The image data taken in tunnels require special treatment, with respect to the cost function formulation, due to the severe radiometric differences in the stereo-pair. These radiometric differences (in exposure and the lighting source position) are caused by the artificial lighting and their amelioration in the matching function is achieved with the use of a modified census transformation on gradients (Stentoumis et al., 2015). In Figure 9 two details of such models are presented.
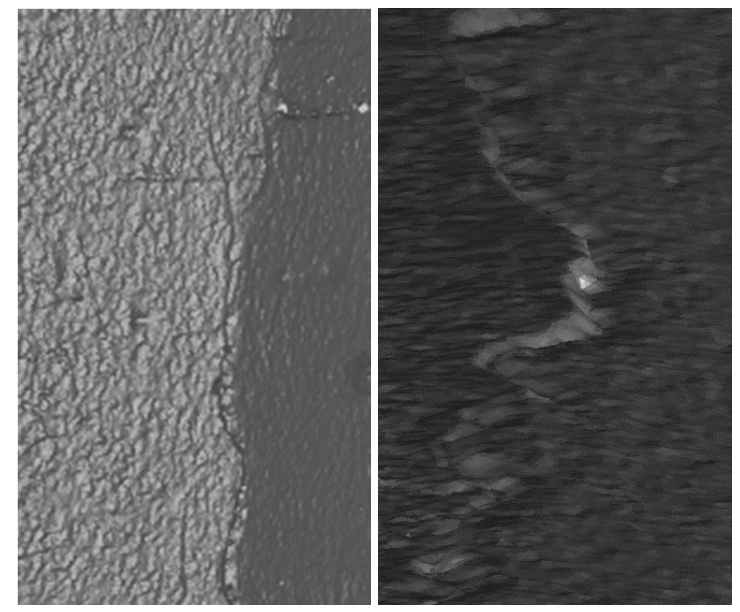

Figure 9. High fidelity 3D models of detected cracks.

\section{LASER SCANNING}

As can be concluded by the laser scanner accuracy values, the laser scanner is unsuitable for the detection of small-sized cracks. However, features that exceed the minimum value of $2 \mathrm{~mm}$ can be detected, e.g. thick cracks, or displacements of the tunnel surface. Moreover, the laser scanner datasets are exploited to extract geometrical features of the tunnel cross-section, which is critical in the detection of possible deformations.

The geometrical shape of a tunnel intrados usually has a quadratic form, e.g. circle, parabola, or an assembly of circular shaped arcs. A nonlinear least-squares algorithm, and particularly the trustregion-reflective method (Coleman and Li, 1996; Coleman and $\mathrm{Li}$, 1994), is utilized to calculate a total number of eight parameters: the parameters $\alpha$ and $\beta$ of the ellipsis; the three rotation parameters; and the three translation parameters. An initial parameter calculation is performed to locate and eliminate outliers, i.e. the points whose distance from the calculated surface exceeds a user defined threshold. The procedure is repeated once again, in order to determine the final surface from the corrected point dataset. Temporal and spatial changes in the tunnel intrados could provide critical information regarding the location and extend of possible deformations.

A small-sized tunnel is used for field measurements. The test tunnel is approximately $60 \mathrm{~m}$ long and $10 \mathrm{~m}$ wide. A number of scans is performed with different combinations of resolution and quality. It should be noted than two independent scans are required to collect data for a complete tunnel cross section. Figure 10 illustrates an aspect of the tunnel, as well as a rough result of the modelled tunnel intrados. The discrepancies between the measured data (purple colour) and the calculated surface (multi-colour surface) are higher at the tunnel base. The use of different geometrical surface, or the assembly of several surfaces will be considered in future research for a more accurate representation of the tunnel inner surface.
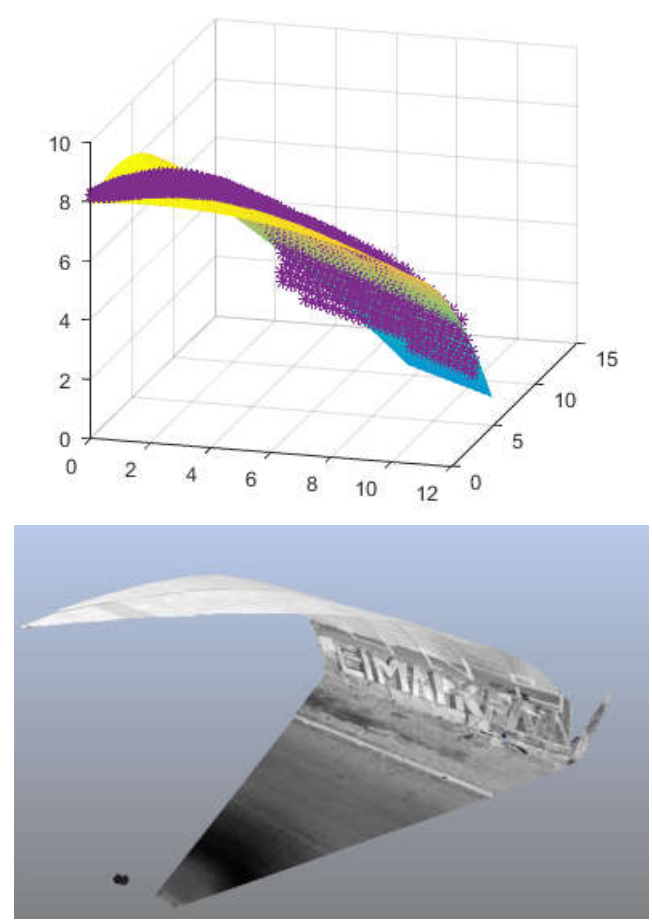

Figure 10: Tested methodology for deformation estimation. Above: the measured data are presented with the purple colour, while the fitting results are illustrated with the multi-colour surface; below: snapshot of the $3 \mathrm{~d}$ point cloud of the test tunnel. 


\section{CONCLUSIONS}

In this work, a working prototype for visual inspection of tunnels is presented. A variety of sensors are used in order to automate the procedure. State-of-the art algorithms in computer vision are exploited in order to detect defects and fully record them in 3D. This will allow the creation of an "inspection base" an archive where the spatio-temporal analysis of the tunnel infrastructure can take place. A lot of work needs to be done in the future before the industry trusts and incorporates such new technologies in the inspection workflow, but the results presented here are promising and automation in inspection is clearly a very active research field.

\section{ACKNOWLEDGEMENTS}

The research leading to these results has received funding from the EC FP7 project ROBO-SPECT (Contract N.611145). Authors wish to thank all partners within the ROBO-SPECT consortium. The writers would like to especially thank M.Sc. Ioannis Damigos from ICCS NTUA team for his dedicated work on the efficient system software implementation.

\section{REFERENCES}

Abdel-Qader, I., Abudayyeh, O., Kelly, M.E., 2003. Analysis of edge-detection techniques for crack identification in bridges. J. Comput. Civ. Eng. 17, pp. 255-263.

Arun, K.S., Huang, T.S., Blostein, S.D., 1987. Least-Squares Fitting of Two 3-D Point Sets. IEEE Trans. Pattern Anal. Mach. Intell. PAMI-9, pp. 698-700.

Coleman, T. F., and Li, Y., 1994. On the convergence of interiorreflective Newton methods for nonlinear minimization subject to bounds. Mathematical programming, 67(1-3), pp. 189-224.

Coleman, T. F., and Li, Y., 1996. An interior trust region approach for nonlinear minimization subject to bounds. SIAM Journal on optimization, 6(2), pp. 418-445.

Douskos, V., Grammatikopoulos, L., Kalisperakis, I., Karras, G., Petsa, E., 2009. FAUCCAL: An open source toolbox for fully automatic camera calibration, in: Proc. XXII CIPA Symposium on Digital Documentation, Interpretation \& Presentation of Cultural Heritage.

Fekete, S., Diederichs, M., Lato, M., 2010. Geotechnical and operational applications for 3-dimensional laser scanning in drill and blast tunnels. Tunn. Undergr. Sp. Technol. 25, pp. 614-628.

Grammatikopoulos, L., Kalisperakis, I., Petsa, E., Stentoumis, C., 2015. 3D city models completion by fusing lidar and image data, in: Proc. SPIE Videometrics. Munich.

Halfawy, M.R., Hengmeechai, J., 2014. Automated defect detection in sewer closed circuit television images using histograms of oriented gradients and support vector machine. Autom. Constr. 38, pp. 1-13.

Hinton, G., Dayan, P., Frey, B., Neal, R., 1995. The “wake-sleep" algorithm for unsupervised neural networks. Science (80), pp. $1158-1161$.

Hirschmüller, H., 2008. Stereo processing by semiglobal matching and mutual information. IEEE Trans. Pattern Anal. Mach. Intell. 30, pp. 328-341.
Jahanshahi, M.R., Masri, S.F., Padgett, C.W., Sukhatme, G.S., 2013. An innovative methodology for detection and quantification of cracks through incorporation of depth perception. Mach. Vis. Appl. 24, pp. 227-241.

Krisada, C., 2014. Damage detection and monitoring for tunnel inspection based on computer vision. University of Cambridge.

Lee, S.Y., Lee, S.H., Shin, D.I., Son, Y.K., Han, C.S., 2007. Development of an inspection system for cracks in a concrete tunnel lining. Can. J. Civ. Eng. 34, pp. 966-975.

Liu, Z., Suandi, S.A., Ohashi, T., Ejima, T., 2002. Tunnel crack detection and classification system based on image processing, in: Electronic Imaging 2002. International Society for Optics and Photonics, pp. 145-152.

Makantasis, K., Protopapadakis, E., Doulamis, A.D., Doulamis, N.D., Loupos, C., 2015. Deep Convolutional Neural Networks for Efficient Vision Based Tunnel Inspection. Cluj-Napoca, Romania.

Paar, G., Caballo-Perucha, M. d. P., Kontrus, H., Sidla, O., 2006. Optical crack following on tunnel surfaces. Proc. SPIE.

Prokos, A., Karras, G., Grammatikopoulos, L., 2009. Design and evaluation of a photogrammetric $3 \mathrm{~d}$ surface scanner, in: XXII CIPA Symposium on Digital Documentation, Interpretation \& Presentation of Cultural Heritage. Kyoto, pp. 1-6.

Protopapadakis, E., Doulamis, N., 2015. Image Based Approaches for Tunnels' Defects Recognition via Robotic Inspectors, in: 11th International Symposium on Visual Computing. To be published, Las Vegas.

Sandrone, F., 2013. Tunnel conditions assessment based on image analysis: A new inspection procedure for railway tunnels, in: Underground - The Way to the Future: Proceedings of the World Tunnel Congress, WTC 2013. pp. 459-465.

Sandrone, F., Labiouse, V., 2011. Identification and analysis of Swiss National Road tunnels pathologies. Tunn. Undergr. Sp. Technol. 26, pp. 374-390.

Sinha, S.K., Fieguth, P.W., 2006. Automated detection of cracks in buried concrete pipe images. Autom. Constr. 15, pp. 58-72.

Son, H., Kim, C., Kim, C., 2012. Automated Color Model-Based Concrete Detection in Construction-Site Images by Using Machine Learning Algorithms. J. Comput. Civ. Eng. 26, pp. 421433.

Metta, G., Fitzpatrick, P., Natale, L., 2006. YARP: Yet Another Robot Platform. International Journal on Advanced Robotics Systems.

Stent, S., Gherardi, R., Bjorn Stenger, Soga, K., Cipolla, R., 2013. An image-based system for change detection on tunnel linings, in: IAPR International Conference on Machine Vision Applications.

Stentoumis, C., Grammatikopoulos, L., Kalisperakis, I., Karras, G., 2014. On accurate dense stereo-matching using a local adaptive multi-cost approach. ISPRS J. Photogramm. Remote Sens. 91, pp. 29-49.

Stentoumis, C., Karras, G., Amditis, A., 2015. Census-based cost on gradients for matching under illumination differences, in: 
IEEE International Conference on 3D Vision. Lyon, pp. 224231.

Victores, J.G., Martínez, S., Jardón, Balaguer, C., 2011. Robotaided tunnel inspection and maintenance system by vision and proximity sensor integration. Autom. Constr. 20, pp. 629-636.

Yoon, J.-S., Sagong, M., Lee, J.S., Lee, K., 2009. Feature extraction of a concrete tunnel liner from 3D laser scanning data. NDT E Int. 42, pp. 97-105.

Yu, S.-N., Jang, J.-H., Han, C.-S., 2007. Auto inspection system using a mobile robot for detecting concrete cracks in a tunnel. Autom. Constr. 16, pp. 255-261.

Yuan, Y., Bai, Y., Liu, J., 2012. Assessment service state of tunnel structure. Tunn. Undergr. Sp. Technol. 27, pp. 72-85. 